\title{
Influence of Non-Genetic Factors on Semen Quality Parameters in Crossbred Jersey (Bos taurus x Bos indicus) Bulls
}

\author{
Alagappan Gopinathan ${ }^{1 *}$, Salem Nagalingam Sivaselvam ${ }^{1}$, \\ Sarojini Kanniappan Karthickeyan ${ }^{1}$ and Ragothaman Venkataramanan ${ }^{2}$
}

${ }^{1}$ Department of Animal Genetics and Breeding, Madras Veterinary College, Chennai, India

${ }^{2}$ Post-Graduate Research Institute in Animal Sciences, Kattupakkam, Tamil Nadu Veterinary and Animal Sciences University, Chennai, India

*Corresponding author

\begin{tabular}{|c|c|}
\hline & A B S T R A C T \\
\hline & \multirow{5}{*}{$\begin{array}{l}\text { Data on } 106197 \text { semen ejaculates of } 310 \text { crossbred Jersey bulls maintained at three frozen } \\
\text { semen stations in Tamil Nadu, India were collected from the year } 2002 \text { to } 2014 \text {. The } \\
\text { effects of various non-genetic factors such as farm, ejaculate number, period of semen } \\
\text { collection, season of semen collection and age of the bulls at collection were studied on } \\
\text { semen quality parameters using least-squares method. The overall least-squares means for } \\
\text { semen volume, sperm concentration, mass activity, initial sperm motility, post-thaw } \\
\text { motility and number of doses per ejaculate were } 4.03 \mathrm{ml}, 1056.18 \text { million per ml, } 2.31 \text {, } \\
67.08 \text { per cent, } 51.04 \text { per cent and } 172.58 \text { doses, respectively. The effects of farm, } \\
\text { ejaculate, period and age of the bulls were significant }(\mathrm{P}<0.05) \text { on all semen quality } \\
\text { parameters. Season had significant }(\mathrm{P}<0.05) \text { effect on sperm concentration, mass activity } \\
\text { and initial sperm motility. The farm x season and age x ejaculate interaction effects were } \\
\text { found to have significant }(\mathrm{P}<0.05) \text { effect on all semen quality parameters. }\end{array}$} \\
\hline & \\
\hline $\begin{array}{l}\text { CBJY bulls, Non- } \\
\text { genetic factors, Semen } \\
\text { production traits }\end{array}$ & \\
\hline Article Info & \\
\hline $\begin{array}{l}\text { Accepted: } \\
\text { 23 March } 2018 \\
\text { Available Online: } \\
10 \text { April } 2018\end{array}$ & \\
\hline
\end{tabular}

\section{Introduction}

In India, artificial insemination programme began around 1951 to improve cattle productivity by crossbreeding the indigenous cattle (Bos indicus) with temperate breeds (Bos taurus). The temperate cattle breeds such as Ayrshire, Brown Swiss, Guernsey, Jersey and Holstein Friesian were crossed with indigenous breeds such as Gir, Sahiwal, Red Sindhi and Tharparkar and non-descript cattle. Over the years, crossbreeding has been adopted by government, quasi-government and private institutions involved in dairy herd improvement activities. Among many exotic breeds used in breeding experiments, Jersey and Holstein Friesian bulls were found to be more suitable for crossbreeding and thus extensively used in various parts of the country. Jersey cattle, by virtue of its higher fat per cent in milk and compact body size, has been preferred as the breed of the choice over Holstein Friesian, which has led to a substantial increase in the population of crossbred Jersey cattle. The breeding policy of India recommends a blood level of 50 per cent exotic inheritance and therefore, central and state governments have given more emphasis 
on producing crossbred Jersey (CBJY) bulls with 50 per cent exotic inheritance for continuous supply of frozen semen for artificial insemination. However, information on the semen production potential of CBJY bulls is limited. The performance of CBJY bulls in sub-tropical climatic condition of southern India has not been evaluated till date. Hence, the present study was carried out to investigate the semen quality parameters of CBJY bulls, to analyse the effect of nongenetic factors on semen quality traits.

\section{Materials and Methods}

Semen production data on 106,197 ejaculates from $310 \mathrm{CBJY}$ bulls during the period from 2002 to 2014 were collected from the records of three frozen semen production stations in Tamil Nadu, India. Two of them, the District Livestock Farm and Nucleus Jersey and Stud Farm are located in hilly Uthagamandalam at an altitude of 2460 to 2662 metres above MSL in the Nilgiris district $\left(11^{\circ} 24^{\prime} \mathrm{N}\right.$ and $\left.76^{\circ} 42^{\prime} \mathrm{E}\right)$ and the third farm, Exotic Cattle Breeding Farm, Eachenkottai is situated in the plains of Thanjavur district at an altitude of 50 metres above MSL $\left(10^{\circ} 45^{\prime} \mathrm{N}\right.$ and $\left.79^{\circ} 29^{\prime} \mathrm{E}\right)$.

The traits chosen for the study were semen volume (SV), sperm concentration (SC), mass activity (MA), initial sperm motility (ISM), post-thaw sperm motility (PTM) and number of doses per ejaculate. The Minimum Standards for Production of Bovine Frozen Semen (2012) of Government of India is followed in all semen production stations for uniform feeding and management of bulls, semen collection, freezing and quality of frozen semen.

The data were analyzed for the effect of nongenetic factors such as farms (F-I, F-II and FIII), ejaculate numbers (E-I and E-II), periods (P-I-2002 to 2004, P-II-2005 to 2007, P-III2008 to 2010 and P-IV-2011 to 2014), seasons
[S-I winter (December, January and February), S-II summer (March, April and May), S-III southwest monsoon (June, July and August) and S-IV northeast monsoon (September, October and November)] and age of the bulls (18 to 42 months, 43 to 67 months, 68 to 92 months, 93 to 117 months, 118 to 142 months and more than 143 months) on semen production.

\section{Statistical analyses}

\section{Effect of non-genetic factors}

To evaluate the effect of non-genetic factors on semen quality parameters, the following fixed general linear model was fitted.

$Y_{i j k l m o}{ }=\mu+F_{i}+E_{j}+P_{k}+S_{l}+A_{m}+\left(F S_{i}\right)_{i l}+$ $(A E)_{m j}+e_{i j k l m o}$

Where, $Y_{i j k l m o}$ is the semen production trait of $\mathrm{o}^{\text {th }}$ observation belonging to $i^{\text {th }}$ farm, $j^{\text {th }}$ ejaculate, $k^{\text {th }}$ period, $l^{\text {th }}$ season and $m^{\text {th }}$ age effects. $\mu$ is the overall mean; $F_{i}$ is the fixed effect of $i^{t h}$ farm ( $i=1$ to 3 ); $\mathrm{E}_{\mathrm{j}}$ is the fixed effect of $j^{\text {th }}$ ejaculate ( $j=1$ and 2); $P_{k}$ is the fixed effect of $k^{\text {th }}$ period ( $k=1$ to 4 ); $S_{l}$ is the fixed effect of $l^{\text {th }}$ season ( $l=1$ to 4$)$ and $A_{m}$ is the fixed effect of $m^{\text {th }}$ age of bull ( $m=1$ to 6); Two-way interactions among effects are represented within parenthesis; $e_{i j k l m o}$ is a residual random error. In this paper, only the effects of farm $x$ season and age $x$ ejaculate interactions were discussed.

The differences between the least-squares means for sub-classes under a particular effect were tested by using Duncan's Multiple Range Test (Kramer, 1957) or Scheffe test (Scheffe, 1959) depending on the heterogeneity of data. The semen quality parameters expressed in percentages such as initial sperm motility and post-thaw motility, were adjusted by angular transformation for statistical analysis. While expressing the means and standard errors, 
angles were reconverted to percentages to a precision of two decimals.

\section{Results and Discussion}

The results of least-squares analysis for various semen production traits and nongenetic factors affecting them are given in Table 1. The interaction effects of these factors were studied and presented in Table 2.

\section{Semen volume}

The overall least-squares means for semen volume was $4.03 \pm 0.01 \mathrm{ml}$ for CBJY bulls. Farm, ejaculate, period and age of the bull contributed significantly $(\mathrm{P}<0.05)$ to the variation in semen volume. Season was not a significant source of variation. The interaction effects such as farm $\mathrm{x}$ season and age $\mathrm{x}$ ejaculate were found to be significant $(\mathrm{P}<0.01)$ on semen volume.

Of the three farms, farm I was in plains and farm II and III were located in hilly area. Bulls reared in farm III donated the highest volume of semen followed by the bulls of farm I and farm II, which could be due to better management of bulls. The reduction in semen volume in the second ejaculate is physiologically normal since the second ejaculate was collected after a very brief resting period of $30 \mathrm{~min}$. Semen volume was found significantly more during period IV (2011-2014) when compared to other periods and this increase in semen volume might be due to improvement in bull management and selection of bulls.

The age of the bull had significant effect on semen volume with older bulls donating significantly more semen than younger bulls. It was also found that CBJY bulls were giving acceptable volume of semen even at the age of 10 to 12 years. Surprisingly, season did not influence the semen volume in CBJY bulls.
However, farm $\mathrm{x}$ season interaction was found significant. In farm I, the highest semen volume was recorded during southwest monsoon $(4.18 \mathrm{ml})$ followed by summer, northeast monsoon and winter seasons. The reason could be better irrigation of fodder crops after rain and good quality fodder availability to bulls. In the hilly area, the highest semen volume was noticed in winter season in farm II and winter and northeast monsoon seasons in farm III. In winter season, the ambient temperature in the hilly area is conducive and the bulls are stress-free leading to better semen production. But during southwest monsoon due to continuous torrential rain throughout the day, the bulls were stressed out could not produce optimum semen volume. However, in summer seasons, non-availability of green fodder and summer stress reduced semen volume. The first ejaculate had higher semen volume than second till 117 months of age, after which second ejaculate had higher semen volume.

Perusal of available literature revealed higher semen volume $(5.53 \mathrm{ml}$, Saxena and Tripathi, 1981; $5.37 \mathrm{ml}$, Jaiswal et al., 1988; $5.51 \mathrm{ml}$, Singh et al., 2000; $4.5 \mathrm{ml}$, Shaha et al., 2008 and $5.25 \mathrm{ml}$, Giri et al., 2011) in CBJY bulls of north India and Bangladesh.

Lower semen volumes were also recorded (3.24 ml, Sagdeo et al., 1990 and $3.56 \mathrm{ml}$, Sagdeo et al., 1992) in CBJY bulls. The differences in semen volume between the present study and earlier studies could be due to the nature of indigenous breed used for crossing with Jersey to produce crossbred bulls. As found in the present study, the season of semen collection did not have any effect on semen volume in an earlier study also (Saxena and Tripathi, 1981). On the contrary, the age of the bulls was found to affect the semen volume in crossbred bulls of Kerala (Mathew et al., 1982 and Sudheer, 2000). 


\section{Sperm concentration}

The least-squares means for sperm concentration was $1056.18 \pm 3.77$ million per $\mathrm{ml}$. Farm, ejaculate, period, season and age of the bull contributed significantly $(\mathrm{P}<0.05)$ to the variation in sperm concentration. The interaction effects such as farm $\mathrm{x}$ season and age $\mathrm{x}$ ejaculate were found to be significant $(\mathrm{P}<0.01)$ on sperm concentration. The highest sperm concentration was seen in farm II followed by farm I and farm III. The first ejaculate had a higher sperm concentration than the second ejaculate. Winter season had the highest sperm concentration followed by the northeast monsoon, southeast monsoon and summer seasons.

The difference in sperm concentrations between seasons might be attributed to variation in green fodder availability, ambient temperature and relative humidity.

Sperm concentration was almost similar from 18 months to 117 months of age with only small variations, but the sperm concentration reduced significantly from 118 months of age. Farm I recorded the highest sperm concentration during winter season as the semen volume was low in winter. But, in farm II and III, the highest sperm concentration was observed in northeast monsoon season during which sufficient green fodder was available and there was comfortable environment to the bulls.

Earlier studies reported higher values (1316 million per ml, Bedi et al., 1984; 1260.7 million per $\mathrm{ml}$, Shaha et al., 2008; 1330 million per ml, Giri et al., 2011) as well as lower sperm concentration (917.85 million per $\mathrm{ml}$, Saxena and Tripathi, 1981; 727.40 million per ml, Singh et al., 2000) in CBJY bulls compared to the present study. Most of the earlier reports revealed significant effect of non-genetic factors such as farm, season, period, age of bull etc. on sperm concentration, while Sudheer (2000) observed a non-significant effect of the age of the bull on sperm concentration.

\section{Mass activity}

The overall least-squares means for mass activity was $2.31 \pm 0.01$. The farm, ejaculate, period season and age of the bull contributed significantly $(\mathrm{P}<0.05)$ to the variation in the mass activity. The mass activity was the highest in farm II followed by farm I and farm III, which was similar in findings of sperm concentration. The interaction effect of farm $x$ season was found to be significant $(\mathrm{P}<0.01)$ on mass activity.

The mass activity is a subjective evaluation and hence there was wide variation between the semen evaluators in different farms and in different periods. The semen collected in winter and southwest monsoon seasons showed the highest mass activity. Growing bulls (18 to 42 months) and older bulls (>143 months) had a lower mass activity than the bulls in other age groups.

Higher mass activity scores of 3.71 (Saxena and Tripathi, 1981), 3.85 (Jaiswal et al., 1988) and 3.83 (Giri et al., 2011) were reported in CBJY bulls by earlier workers. On the other hand, lower scores of 1.93 and 1.77 (Sagdeo et al., 1990 and 1992) were also reported. To the best of knowledge, no Indian literature is available to compare the effects of various non-genetic factors on mass activity in CBJY bulls.

\section{Initial sperm motility}

The overall least-squares means for initial sperm motility was $67.08 \pm 0.00$ per cent. The farm, ejaculate, period, season and age of the bull contributed significantly $(\mathrm{P}<0.05)$ to the variation in initial sperm motility. 
Table.1 Least-squares means $( \pm$ SEM) for semen quality parameters in crossbred Jersey bulls

\begin{tabular}{|c|c|c|c|c|c|c|}
\hline Effects & $\begin{array}{c}\text { Semen } \\
\text { volume (ml) }\end{array}$ & $\begin{array}{c}\text { Sperm } \\
\text { concentration } \\
\text { (millions per } \mathbf{m l} \text { ) }\end{array}$ & $\begin{array}{l}\text { Mass activity } \\
(0 \text { to } 5 \text { scale) }\end{array}$ & $\begin{array}{l}\text { Initial sperm } \\
\text { motility } \\
\text { (per cent) }\end{array}$ & $\begin{array}{l}\text { Post-thaw } \\
\text { motility } \\
\text { (per cent) }\end{array}$ & $\begin{array}{l}\text { No. of doses } \\
\text { per ejaculate }\end{array}$ \\
\hline Overall & $\begin{array}{c}4.03 \pm 0.01 \\
(106197)\end{array}$ & $\begin{array}{c}1056.18 \pm 3.77 \\
(106197)\end{array}$ & $\begin{array}{l}2.31 \pm 0.01 \\
(106197)\end{array}$ & $\begin{array}{c}67.08 \pm 0.00 \\
(106197)\end{array}$ & $\begin{array}{c}51.04 \pm 0.00 \\
(74638)\end{array}$ & $\begin{array}{c}172.58 \pm 0.94 \\
(73334)\end{array}$ \\
\hline Farm & $*$ & $*$ & $*$ & $*$ & $*$ & $*$ \\
\hline I & $\begin{array}{c}4.08^{\mathrm{a}} \pm 0.01 \\
(33784)\end{array}$ & $\begin{array}{c}1001.67^{b} \pm 4.63 \\
(33784)\end{array}$ & $\begin{array}{c}1.96^{\mathrm{b}} \pm 0.01 \\
(33784)\end{array}$ & $\begin{array}{c}68.97^{b} \pm 0.00 \\
(33784)\end{array}$ & $\begin{array}{c}53.14^{\mathrm{a}} \pm 0.00 \\
(26947)\end{array}$ & $\begin{array}{c}153.26^{\mathrm{c}} \pm 1.17 \\
(26838)\end{array}$ \\
\hline II & $\begin{array}{c}3.78^{b} \pm 0.02 \\
(11492)\end{array}$ & $\begin{array}{c}1202.71^{\mathrm{a}} \pm 5.75 \\
(11492)\end{array}$ & $\begin{array}{c}3.42^{\mathrm{a}} \pm 0.01 \\
(11492)\end{array}$ & $\begin{array}{c}78.87^{\mathrm{a}} \pm 0.00 \\
(11492)\end{array}$ & $\begin{array}{c}50.94^{b} \pm 0.00 \\
(10741)\end{array}$ & $\begin{array}{c}153.30^{\mathrm{b}} \pm 1.36 \\
(10466)\end{array}$ \\
\hline III & $\begin{array}{c}4.23^{\mathrm{a}} \pm 0.01 \\
(60921)\end{array}$ & $\begin{array}{c}964.16^{\mathrm{c}} \pm 3.67 \\
(60921)\end{array}$ & $\begin{array}{c}1.53^{c} \pm 0.01 \\
(60921)\end{array}$ & $\begin{array}{c}52.05^{\mathrm{c}} \pm 0.00 \\
(60921)\end{array}$ & $\begin{array}{c}49.04^{c} \pm 0.00 \\
(36950)\end{array}$ & $\begin{array}{c}211.18^{\mathrm{a}} \pm 0.97 \\
(36030)\end{array}$ \\
\hline Ejaculate & $*$ & $*$ & $*$ & $*$ & $*$ & $*$ \\
\hline I & $\begin{array}{c}4.09 \pm 0.02 \\
(58419)\end{array}$ & $\begin{array}{c}1119.71 \pm 4.98 \\
(58419)\end{array}$ & $\begin{array}{l}2.33 \pm 0.01 \\
(58419)\end{array}$ & $\begin{array}{c}65.03 \pm 0.00 \\
(58419)\end{array}$ & $\begin{array}{c}47.16 \pm 0.00 \\
(38415)\end{array}$ & $\begin{array}{c}192.65 \pm 1.26 \\
(38152)\end{array}$ \\
\hline II & $\begin{array}{c}3.97 \pm 0.02 \\
(47778)\end{array}$ & $\begin{array}{c}992.66 \pm 5.08 \\
(47778)\end{array}$ & $\begin{array}{c}2.28 \pm 0.01 \\
\quad(47778)\end{array}$ & $\begin{array}{c}69.10 \pm 0.00 \\
(47778)\end{array}$ & $\begin{array}{c}51.43 \pm 0.00 \\
(36223)\end{array}$ & $\begin{array}{c}152.51 \pm 1.25 \\
(35182)\end{array}$ \\
\hline Period & $*$ & $*$ & $*$ & $*$ & $*$ & $*$ \\
\hline I (2002 - 2004) & $\begin{array}{c}3.75^{\mathrm{c}} \pm 0.02 \\
(20188)\end{array}$ & $\begin{array}{c}986.16^{\mathrm{d}} \pm 5.02 \\
(20188)\end{array}$ & $\begin{array}{c}2.40^{\mathrm{b}} \pm 0.01 \\
(20188)\end{array}$ & $\begin{array}{c}68.89^{\mathrm{a}} \pm 0.00 \\
(20188)\end{array}$ & $\begin{array}{c}51.52^{\mathrm{a}} \pm 0.00 \\
(14265)\end{array}$ & $\begin{array}{c}141.01^{c} \pm 1.24 \\
(14236)\end{array}$ \\
\hline II (2005 - 2007) & $\begin{array}{c}3.89^{c} \pm 0.02 \\
(19517)\end{array}$ & $\begin{array}{c}1045.81^{c} \pm 5.42 \\
(19517)\end{array}$ & $\begin{array}{c}2.21^{c} \pm 0.01 \\
\quad(19517)\end{array}$ & $\begin{array}{c}68.80^{\mathrm{a}} \pm 0.00 \\
(19517)\end{array}$ & $\begin{array}{c}50.98^{c} \pm 0.00 \\
(11921)\end{array}$ & $\begin{array}{c}160.17^{b} \pm 1.42 \\
(11873)\end{array}$ \\
\hline III (2008 - 2010) & $\begin{array}{c}4.06^{b} \pm 0.01 \\
(33119)\end{array}$ & $\begin{array}{c}1110.96^{\mathrm{a}} \pm 4.42 \\
(33119)\end{array}$ & $\begin{array}{c}2.15^{\mathrm{d}} \pm 0.01 \\
(33119)\end{array}$ & $\begin{array}{c}65.91^{b} \pm 0.00 \\
(33119)\end{array}$ & $\begin{array}{c}51.12^{b} \pm 0.00 \\
(21803)\end{array}$ & $\begin{array}{c}177.01^{\mathrm{b}} \pm 1.13 \\
(21790)\end{array}$ \\
\hline
\end{tabular}




\begin{tabular}{|c|c|c|c|c|c|c|}
\hline IV (2011 - 2014) & $\begin{array}{c}4.41^{\mathrm{a}} \pm 0.01 \\
(33373)\end{array}$ & $\begin{array}{c}1081.79^{b} \pm 4.20 \\
(33373)\end{array}$ & $\begin{array}{c}2.47^{\mathrm{a}} \pm 0.01 \\
(33373)\end{array}$ & $\begin{array}{c}64.66^{c} \pm 0.00 \\
(33373)\end{array}$ & $\begin{array}{c}50.56^{\mathrm{d}} \pm 0.00 \\
(26649)\end{array}$ & $\begin{array}{c}212.13^{\mathrm{a}} \pm 1.08 \\
(25435)\end{array}$ \\
\hline Season & NS & $*$ & $*$ & $*$ & NS & NS \\
\hline Winter & $\begin{array}{c}4.08 \pm 0.02 \\
(26240)\end{array}$ & $\begin{array}{c}1085.40^{\mathrm{a}} \pm 6.27 \\
\quad(26240)\end{array}$ & $\begin{array}{c}2.38^{\mathrm{a}} \pm 0.01 \\
(26240)\end{array}$ & $\begin{array}{c}67.99^{a} \pm 0.00 \\
(26240)\end{array}$ & $\begin{array}{c}51.13 \pm 0.00 \\
(18914)\end{array}$ & $\begin{array}{c}174.64 \pm 1.47 \\
(18554)\end{array}$ \\
\hline Summer & $\begin{array}{c}4.01 \pm 0.02 \\
(26342)\end{array}$ & $\begin{array}{c}1023.84^{c} \pm 7.10 \\
(26342)\end{array}$ & $\begin{array}{c}2.32^{b} \pm 0.01 \\
(26342)\end{array}$ & $\begin{array}{c}65.98^{c} \pm 0.00 \\
(26342)\end{array}$ & $\begin{array}{c}50.99 \pm 0.00 \\
(18074)\end{array}$ & $\begin{array}{c}171.29 \pm 1.85 \\
(17950)\end{array}$ \\
\hline Southwest monsoon & $\begin{array}{c}3.99 \pm 0.03 \\
(27397)\end{array}$ & $\begin{array}{c}1053.19^{c} \pm 8.37 \\
(27397)\end{array}$ & $\begin{array}{c}2.39^{\mathrm{a}} \pm 0.01 \\
(27397)\end{array}$ & $\begin{array}{c}66.52^{c} \pm 0.00 \\
\quad(27397)\end{array}$ & $\begin{array}{c}50.92 \pm 0.00 \\
(19018)\end{array}$ & $\begin{array}{c}172.47 \pm 2.19 \\
(18820)\end{array}$ \\
\hline Northeast monsoon & $\begin{array}{c}4.04 \pm 0.02 \\
\quad(26218)\end{array}$ & $\begin{array}{c}1062.29^{b} \pm 7.41 \\
(26218)\end{array}$ & $\begin{array}{l}2.13^{c} \pm 0.01 \\
\quad(26218)\end{array}$ & $\begin{array}{c}67.80^{\mathrm{b}} \pm 0.00 \\
(26218)\end{array}$ & $\begin{array}{c}51.11 \pm 0.00 \\
\quad(18632)\end{array}$ & $\begin{array}{c}171.91 \pm 1.74 \\
(18010)\end{array}$ \\
\hline Age & $*$ & $*$ & $*$ & $*$ & $*$ & $*$ \\
\hline I $(18$ to $42 \mathrm{~m})$ & $\begin{array}{c}3.08^{\mathrm{e}} \pm 0.01 \\
(32977)\end{array}$ & $\begin{array}{c}1112.16^{\mathrm{a}} \pm 3.03 \\
(32977)\end{array}$ & $\begin{array}{c}2.26^{\mathrm{b}} \pm 0.01 \\
(32977)\end{array}$ & $\begin{array}{c}59.64^{\mathrm{d}} \pm 0.00 \\
(32977)\end{array}$ & $\begin{array}{c}51.51^{\mathrm{a}} \pm 0.00 \\
(20414)\end{array}$ & $\begin{array}{c}163.54^{\mathrm{c}} \pm 0.78 \\
(20169)\end{array}$ \\
\hline II (43 to $67 \mathrm{~m})$ & $\begin{array}{c}3.62^{d} \pm 0.01 \\
(43763)\end{array}$ & $\begin{array}{c}1099.11^{b} \pm 2.80 \\
(43763)\end{array}$ & $\begin{array}{c}2.32^{\mathrm{a}} \pm 0.01 \\
(43763)\end{array}$ & $\begin{array}{c}66.21^{c} \pm 0.00 \\
(43763)\end{array}$ & $\begin{array}{c}51.53^{\mathrm{ab}} \pm 0.00 \\
(31860)\end{array}$ & $\begin{array}{c}173.21^{b} \pm 0.69 \\
(31455)\end{array}$ \\
\hline III (68 to 92 m) & $\begin{array}{c}4.15^{\mathrm{c}} \pm 0.01 \\
\quad(18672)\end{array}$ & $\begin{array}{c}1101.61^{\mathrm{b}} \pm 4.07 \\
(18672)\end{array}$ & $\begin{array}{c}2.39^{\mathrm{a}} \pm 0.01 \\
\quad(18672)\end{array}$ & $\begin{array}{c}68.76^{\mathrm{b}} \pm 0.00 \\
(18672)\end{array}$ & $\begin{array}{c}51.27^{b c} \pm 0.00 \\
(14133)\end{array}$ & $\begin{array}{c}189.09^{\mathrm{a}} \pm 1.00 \\
(13822)\end{array}$ \\
\hline IV (93 to 117 m) & $\begin{array}{c}4.32^{b} \pm 0.02 \\
(7013)\end{array}$ & $\begin{array}{c}1095.92^{b} \pm 6.25 \\
(7013)\end{array}$ & $\begin{array}{c}2.39^{\mathrm{a}} \pm 0.01 \\
(7013)\end{array}$ & $\begin{array}{c}68.14^{\mathrm{bc}} \pm 0.00 \\
(7013)\end{array}$ & $\begin{array}{c}51.13^{c} \pm 0.00 \\
(5241)\end{array}$ & $\begin{array}{c}191.41^{\mathrm{a}} \pm 1.52 \\
(5029)\end{array}$ \\
\hline $\mathrm{V}(118$ to $142 \mathrm{~m})$ & $\begin{array}{c}4.52^{\mathrm{a}} \pm 0.03 \\
(2851)\end{array}$ & $\begin{array}{c}1000.48^{c} \pm 9.37 \\
(2851)\end{array}$ & $\begin{array}{c}2.36^{\mathrm{a}} \pm 0.02 \\
(2851)\end{array}$ & $\begin{array}{c}72.69^{\mathrm{a}} \pm 0.00 \\
(2851)\end{array}$ & $\begin{array}{c}51.10^{\mathrm{d}} \pm 0.00 \\
(2313)\end{array}$ & $\begin{array}{c}164.53^{\mathrm{b}} \pm 2.22 \\
(2206)\end{array}$ \\
\hline $\mathrm{VI}(>143 \mathrm{~m})$ & $\begin{array}{c}4.47^{\mathrm{ab}} \pm 0.05 \\
(921)\end{array}$ & $\begin{array}{c}927.81^{c} \pm 16.91 \\
(921)\end{array}$ & $\begin{array}{c}2.11^{b} \pm 0.03 \\
(921)\end{array}$ & $\begin{array}{c}66.67^{c} \pm 0.00 \\
(921)\end{array}$ & $\begin{array}{c}49.72^{\mathrm{e}} \pm 0.00 \\
(677)\end{array}$ & $\begin{array}{c}153.70^{\mathrm{d}} \pm 4.45 \\
(653)\end{array}$ \\
\hline
\end{tabular}

* -Significant $(\mathrm{P}<0.05)$ and NS- Non-significant. Figures in parentheses indicate number of observations.

Means with at least one common superscript within classes do not differ significantly $(\mathrm{P}>0.05)$ 
Table.2 Least-squares means $( \pm$ SEM) for interactions of non-genetic factors on semen quality parameters in crossbred Jersey bulls

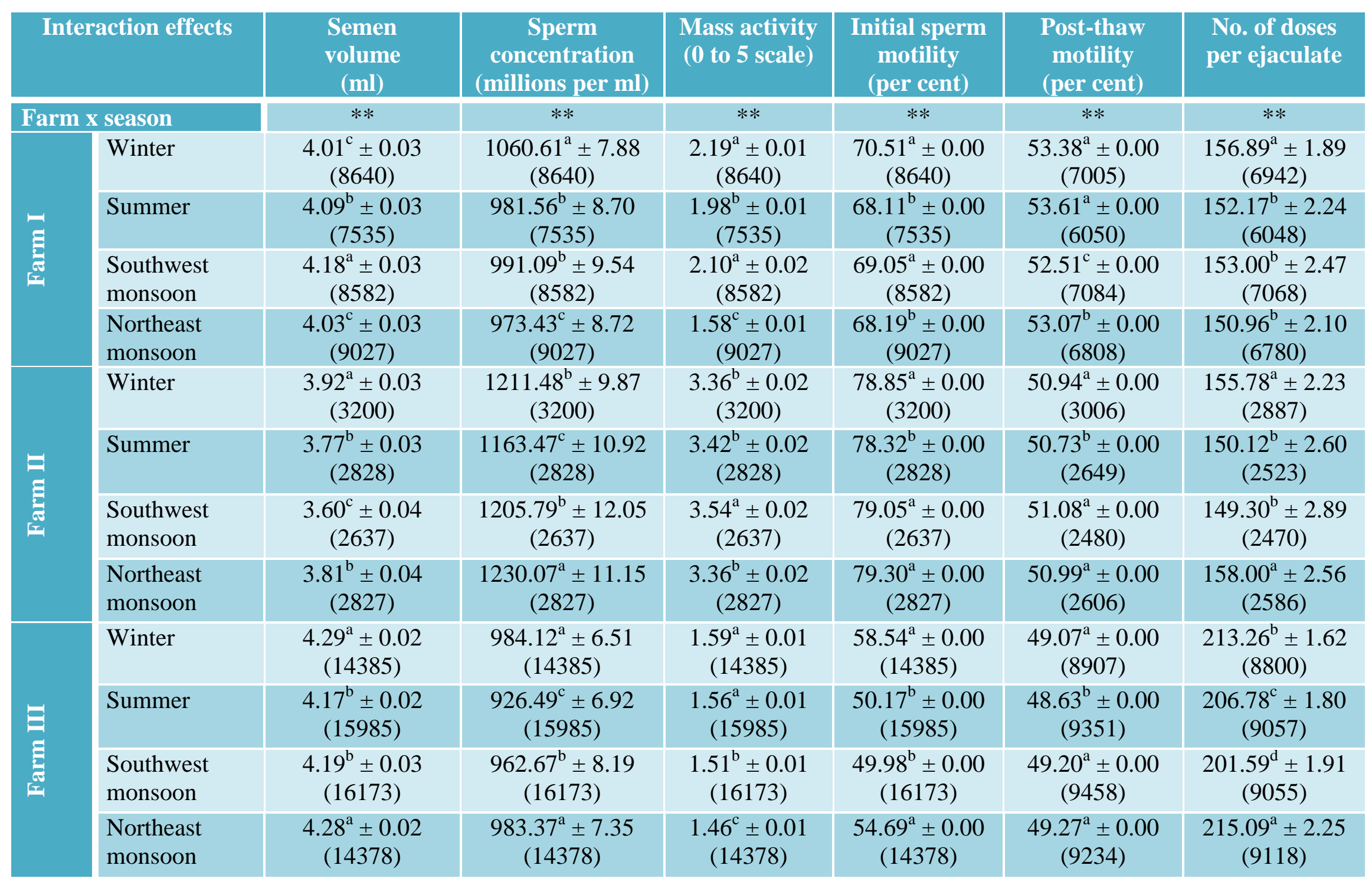




\begin{tabular}{|c|c|c|c|c|c|c|c|}
\hline \multicolumn{2}{|c|}{ Interaction effects } & $\begin{array}{l}\text { Semen } \\
\text { volume } \\
\text { (ml) }\end{array}$ & $\begin{array}{c}\text { Sperm } \\
\text { concentration } \\
\text { (millions per } \mathbf{m l})\end{array}$ & $\begin{array}{l}\text { Mass activity } \\
(0 \text { to } 5 \text { scale) }\end{array}$ & $\begin{array}{l}\text { Initial sperm } \\
\text { motility } \\
\text { (per cent) }\end{array}$ & $\begin{array}{l}\text { Post-thaw } \\
\text { motility } \\
\text { (per cent) }\end{array}$ & $\begin{array}{l}\text { No. of doses } \\
\text { per ejaculate }\end{array}$ \\
\hline \multicolumn{2}{|c|}{ Age $x$ ejaculate } & ** & $* *$ & NS & $* *$ & $* *$ & $* *$ \\
\hline \multirow{2}{*}{ 赵 } & I ejaculate & $\begin{array}{l}3.31 \pm 0.01 \\
(20489)\end{array}$ & $\begin{array}{c}1185.01 \pm 3.61 \\
(20489)\end{array}$ & $\begin{array}{l}2.28 \pm 0.01 \\
(20489)\end{array}$ & $\begin{array}{l}56.40 \pm 0.00 \\
(20489)\end{array}$ & $\begin{array}{l}51.01 \pm 0.00 \\
\quad(11683)\end{array}$ & $\begin{array}{c}190.06 \pm 0.99 \\
(11550)\end{array}$ \\
\hline & II ejaculate & $\begin{array}{l}2.85 \pm 0.01 \\
(12488)\end{array}$ & $\begin{array}{c}1039.30 \pm 4.55 \\
(12488)\end{array}$ & $\begin{array}{l}2.23 \pm 0.01 \\
(12488)\end{array}$ & $\begin{array}{l}62.82 \pm 0.03 \\
\quad(12488)\end{array}$ & $\begin{array}{l}52.02 \pm 0.00 \\
(8731)\end{array}$ & $\begin{array}{c}137.01 \pm 1.15 \\
(8619)\end{array}$ \\
\hline \multirow{2}{*}{ 是 } & I ejaculate & $\begin{array}{l}3.72 \pm 0.01 \\
\quad(22816)\end{array}$ & $\begin{array}{c}1176.02 \pm 3.57 \\
(22816)\end{array}$ & $\begin{array}{l}2.36 \pm 0.01 \\
(22816)\end{array}$ & $\begin{array}{l}64.08 \pm 0.00 \\
\quad(22816)\end{array}$ & $\begin{array}{l}51.26 \pm 0.00 \\
\quad(15628)\end{array}$ & $\begin{array}{c}194.64 \pm 0.91 \\
(15423)\end{array}$ \\
\hline & II ejaculate & $\begin{array}{l}3.51 \pm 0.01 \\
(20947)\end{array}$ & $\begin{array}{c}1022.20 \pm 3.72 \\
(20947)\end{array}$ & $\begin{array}{l}2.29 \pm 0.01 \\
(20947)\end{array}$ & $\begin{array}{l}68.31 \pm 0.00 \\
\quad(20947)\end{array}$ & $\begin{array}{l}57.81 \pm 0.00 \\
\quad(16232)\end{array}$ & $\begin{array}{c}151.78 \pm 0.91 \\
(16032)\end{array}$ \\
\hline \multirow{2}{*}{ 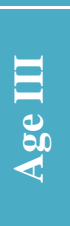 } & I ejaculate & $\begin{array}{l}4.21 \pm 0.01 \\
\quad(9583)\end{array}$ & $\begin{array}{c}1187.98 \pm 5.35 \\
(9583)\end{array}$ & $\begin{array}{l}2.42 \pm 0.01 \\
\quad(9583)\end{array}$ & $\begin{array}{c}66.82 \pm 0.00 \\
(9583)\end{array}$ & $\begin{array}{l}50.84 \pm 0.00 \\
\quad(6881)\end{array}$ & $\begin{array}{c}209.98 \pm 1.35 \\
(6671)\end{array}$ \\
\hline & II ejaculate & $\begin{array}{l}4.10 \pm 0.02 \\
\quad(9089)\end{array}$ & $\begin{array}{c}1015.23 \pm 5.48 \\
(9089)\end{array}$ & $\begin{array}{c}2.36 \pm 0.01 \\
(9089)\end{array}$ & $\begin{array}{c}70.69 \pm 0.00 \\
(9089)\end{array}$ & $\begin{array}{c}51.69 \pm 0.00 \\
(7252)\end{array}$ & $\begin{array}{c}168.20 \pm 1.32 \\
(7151)\end{array}$ \\
\hline \multirow{2}{*}{$\underset{8}{80}$} & I ejaculate & $\begin{array}{c}4.36 \pm 0.03 \\
\quad(3577)\end{array}$ & $\begin{array}{c}1165.66 \pm 8.43 \\
(3577)\end{array}$ & $\begin{array}{l}2.41 \pm 0.01 \\
(3577)\end{array}$ & $\begin{array}{c}66.59 \pm 0.00 \\
(3577)\end{array}$ & $\begin{array}{l}50.82 \pm 0.00 \\
(2570)\end{array}$ & $\begin{array}{c}214.59 \pm 2.11 \\
(2515)\end{array}$ \\
\hline & II ejaculate & $\begin{array}{l}4.28 \pm 0.03 \\
\quad(3436)\end{array}$ & $\begin{array}{c}1026.17 \pm 8.65 \\
(3436)\end{array}$ & $\begin{array}{l}2.36 \pm 0.01 \\
\quad(3436)\end{array}$ & $\begin{array}{c}69.70 \pm 0.00 \\
(3436)\end{array}$ & $\begin{array}{c}51.45 \pm 0.00 \\
(2671)\end{array}$ & $\begin{array}{c}168.23 \pm 2.08 \\
(2514)\end{array}$ \\
\hline \multirow{2}{*}{$\underset{0}{\infty}$} & I ejaculate & $\begin{array}{c}4.48 \pm 0.04 \\
\quad(1470)\end{array}$ & $\begin{array}{c}1048.49 \pm 12.88 \\
(1470)\end{array}$ & $\begin{array}{c}2.36 \pm 0.02 \\
(1470)\end{array}$ & $\begin{array}{c}70.48 \pm 0.00 \\
(1470)\end{array}$ & $\begin{array}{l}50.89 \pm 0.00 \\
(1127)\end{array}$ & $\begin{array}{c}177.95 \pm 3.14 \\
(1105)\end{array}$ \\
\hline & II ejaculate & $\begin{array}{l}4.56 \pm 0.04 \\
\quad(1381)\end{array}$ & $\begin{array}{c}952.47 \pm 13.28 \\
(1381)\end{array}$ & $\begin{array}{c}2.35 \pm 0.02 \\
(1381)\end{array}$ & $\begin{array}{c}74.88 \pm 0.00 \\
(1381)\end{array}$ & $\begin{array}{c}51.29 \pm 0.00 \\
(1186)\end{array}$ & $\begin{array}{c}151.11 \pm 3.07 \\
(1101)\end{array}$ \\
\hline \multirow{2}{*}{$\underset{8}{8}$} & I ejaculate & $\begin{array}{l}4.45 \pm 0.08 \\
\quad(464)\end{array}$ & $\begin{array}{c}955.06 \pm 23.22 \\
(464)\end{array}$ & $\begin{array}{c}2.17 \pm 0.04 \\
(464)\end{array}$ & $\begin{array}{c}65.45 \pm 0.01 \\
(464)\end{array}$ & $\begin{array}{c}49.11 \pm 0.00 \\
(326)\end{array}$ & $\begin{array}{c}168.69 \pm 6.11 \\
(320)\end{array}$ \\
\hline & II ejaculate & $\begin{array}{l}4.50 \pm 0.08 \\
\quad(457)\end{array}$ & $\begin{array}{c}900.55 \pm 23.42 \\
(457)\end{array}$ & $\begin{array}{c}2.06 \pm 0.04 \\
(457)\end{array}$ & $\begin{array}{c}67.88 \pm 0.01 \\
(457)\end{array}$ & $\begin{array}{c}50.33 \pm 0.00 \\
(351)\end{array}$ & $\begin{array}{c}138.72 \pm 5.93 \\
(333)\end{array}$ \\
\hline
\end{tabular}

** - Highly significant $(\mathrm{P}<0.01)$ and NS- Non-significant. Figures in parentheses indicate number of observations.

Means with at least one common superscript within classes do not differ significantly $(\mathrm{P}>0.05)$ 
The interaction effects such as farm $\mathrm{x}$ season and age $x$ ejaculate were found to be significant $(\mathrm{P}<0.01)$ on initial sperm motility. The highest initial sperm motility was observed in farm II, ejaculate II, periods I and II, winter season and age group V.

The estimation of initial sperm motility is also a subjective evaluation and therefore, subjective variations between assessments are bound to happen. The second ejaculate exhibited higher initial sperm motility because it had higher sperm concentration and less proportion of dead sperms. In farm I, the bulls had significantly higher initial sperm motility in winter and southwest monsoon when compared to other seasons. In farm II, the highest per cent initial sperm motility was observed in northeast monsoon, followed by southwest and winter seasons, but, the differences in values between them were not statistically different. In farm III, winter and northeast monsoon seasons produced semen with maximum sperm motility.

The estimates were within the range of estimates reported earlier for CBJY bulls. Higher initial sperm motility estimates of 70.46 per cent (Saxena and Tripathi, 1981), 72.70 per cent (Jaiswal et al., 1988), 79.95 per cent (Singh et al., 2000) and 75.60 per cent (Giri et al., 2011) or lower initial sperm motility per cent of 37.24 and 35.50 (Sagdeo et al., 1990 and 1992) and 60.2 (Shaha et al., 2008) were observed from the literature.

\section{Post-thaw motility}

The overall least-squares means for post-thaw motility was $51.04 \pm 0.00$ per cent. The farm, ejaculate, period and age of the bull contributed significantly $(\mathrm{P}<0.05)$ to the variation in post-thaw motility. Season was not a significant source of variation for postthaw motility. The interaction effects such as farm $x$ season and age $\mathrm{x}$ ejaculate were found to be significant $(\mathrm{P}<0.05)$ on post-thaw motility. Surprisingly, all earlier works reported lower post-thaw motility per cent than that of the present study [43.33 by Tuli et al., (1988), 42.65 and 40.13 by Sagdeo et al., (1990 and 1992), 23.65 by Nair (1997) and 48.12 by Giri et al., (2011)].

Farm I had the highest post-thaw motility followed by farm II and farm III. Similar to the initial sperm motility, higher post-thaw motility was seen in the frozen semen produced from the second ejaculate. The age of the bull was also a significant source of variation for the post-thaw motility with highest value seen in growing and younger bulls. The oldest bulls (>143 months) had the lowest post-thaw motility of 49.72 per cent. In farm I, the highest values were observed during winter and summer seasons, but those values did not differ significantly. In farms II and III, no significant differences were observed in post-thaw motility between northeast, southwest and winter seasons, while it was the lowest in summer season. The higher post-thaw motility values obtained in this study could be due to higher selection pressure imposed on breeding bulls and better freezing and thawing protocols followed by the frozen semen stations.

\section{Number of frozen semen doses per ejaculate}

The overall least-squares mean number of frozen semen doses per ejaculate was 172.58 \pm 0.94 . The effects of farm, ejaculate, period and age of the bull were significant $(\mathrm{P}<0.05)$ on the number of doses produced per ejaculate, while season was not a significant source of variation for the trait. The interaction effects such as farm $\mathrm{x}$ season and age $x$ ejaculate were found to be significant $(\mathrm{P}<0.01)$ on number of doses per ejaculate. As there is dearth of literature on the number of doses of frozen semen produced per ejaculate 
in CBJY bulls, the results obtained in the present study could not be compared.

Farm III produced the highest doses of frozen semen per ejaculate than the other two farms. During northeast monsoon season, maximum doses per ejaculate were produced in both the farms located in hilly area while it was during winter season in the farm located in the plains.

The present study is the first of its kind in India on crossbred Jersey bulls wherein semen quality parameters is assessed based on large number of data along with repeatability and phenotypic correlations. These findings would be useful for the planners and administrators for forecasting the requirement of CBJY bulls for frozen semen stations and continuous supply of frozen semen doses of CBJY bulls for artificial insemination.

\section{Acknowledgement}

The authors express their gratitude to the Tamil Nadu Veterinary and Animal Sciences University granting for permission to pursue this work. The authors are also thankful to the Director, Animal Husbandry and Veterinary Services and the Managing Director, Tamil Nadu Co-operative Milk Producers' Federation Limited for according permission to utilize the semen production data from the frozen semen stations.

\section{References}

Bedi, K.S., Biswas, R.K. and Durgwekar, Y.G. 1984. Fructolysis in semen and its relation to breeding performance in buffalo bull. Indian J. Anim. Reprod., 4: 60-63.

Duncan, D.B. 1955. Multiple range and multiple F-tests. Biometrics, 11:1-42.

Giri, D.K., Barik, A.K., Mohanty, D.N., Mishra, P.C., Tripathy, A.K., Palai,
T.K. and Rath, T.K. 2011. Semen characteristics and conception rate in crossbred Jersey at different thawing temperatures. Indian J. Field Veterinarians, 6: 73-75.

Jaiswal, H.L., Saxena, V.B. and Tripathi, S.S. 1988. Physico-morphological characters of semen and their interrelationships in crossbred bulls. Indian Vet J., 65: 266267.

Meyer, K. 2007. WOMBAT-A tool for mixed model analyses in quantitative genetics by restricted maximum likelihood (REML). J. Zhejiang Uni. Sci., 8: 815821.

Minimum Standards for Production (MSP) of Bovine Frozen Semen, 2012. Published by Department of Animal Husbandry, Dairying and Fisheries, Ministry of Agriculture, Government of India. (http://dahd.nic.in)

Patel, J.B., Dhami, A.J. and Patel, P.A. 2012. Comparative evaluation of physiomorphological attributes of semen of Jafarabadi, Mehsana and crossbred (Kankrej X HF) bulls. Indian J. Field Veterinarians, 7: 1-7.

Pearson, K. 1920. Notes on the history of correlation. Biometrika 13: 25-45.

Raja, C.K.S.V. and Rao, A.R. 1983. Semen characteristics of Brown Swiss crossbred bulls. Indian Vet. J., 60: 2328.

Sagdeo, L.R., Chitnis, A.B., Deshmukh, S.N. and Kaikini, A.S. 1992. Semen freezability in relation to single or multiple exotic genetic components in crossbred bulls. Indian J. Anim. Reprod., 13: 131-133.

Sagdeo, L.R., Chitnis, A.B., Deshmukh, S.N. and Kaikini, A.S. 1990. Studies on semen freezability of pure Jersey with varying levels of exotic inheritance. Indian J. Anim. Reprod., 11: 79-84.

Saxena, V.B. and Tripathi, S.S. 1981. Seasonal variation in semen 
characteristics and preservability in crossbred bulls. Indian J. Anim. Sci., 15: $19-24$

Scheffé, H. 1959. The Analysis of Variance. Wiley, New York, USA.

Shaha, S.P., Alam, M.G.S., Khatun, M. and Ahmed, J.U. 2008. Breeding soundness of stud bulls. The Bangladesh Veterinarian, 25: 51-61.

Singh, S.P., Pandit, R.K. and Bhadoria, H.B.S. 2000. Sexual behavior and seminal characteristics in Jersey, Sahiwal and Half-bred bulls. Indian J. Anim. Sci., 70: 279-280.
Snedecor, G.W. and Cochran, W.G. 1987. Statistical Methods. $5^{\text {th }}$ ed. Iowa State University Press: USA.

Sudheer, S. 2000. Relationship of age and scrotal circumference with the seminal characteristics of crossbred bulls. Indian J. Anim. Sci., 70: 804-806.

Tuli, R.K., Batabyal, A.K., Singal, S.P. and Razdan, M.N. 1988. Seminal characteristics and deep freezing of crossbred bull semen using Tri yolk glycerol extender. Indian J. Anim. Reprod., 9: 16-18.

\section{How to cite this article:}

Alagappan Gopinathan, Salem Nagalingam Sivaselvam, Sarojini Kanniappan Karthickeyan and Ragothaman Venkataramanan. 2018. Influence of Non-Genetic Factors on Semen Quality Parameters in Crossbred Jersey (Bos taurus x Bos indicus) Bulls. Int.J.Curr.Microbiol.App.Sci. 7(04): 2994-3004. doi: https://doi.org/10.20546/ijcmas.2018.704.339 